\title{
Supplementary material: The Mediterranean climate change hotspot in the CMIP5 and CMIP6 projections
}

Josep $\operatorname{Cos}^{1}$, Francisco Doblas-Reyes ${ }^{1,2}$, Martin Jury ${ }^{1}$, Raül Marcos ${ }^{1}$, Pière-Antoine Bretonnière ${ }^{1}$, and Margarida Samsó ${ }^{1}$

${ }^{1}$ Earth Sciences Department, Barcelona Supercomputing Center (BSC), Barcelona, Spain.

${ }^{2}$ Institució Catalana de Recerca i Estudis Avançats (ICREA), Barcelona, Spain

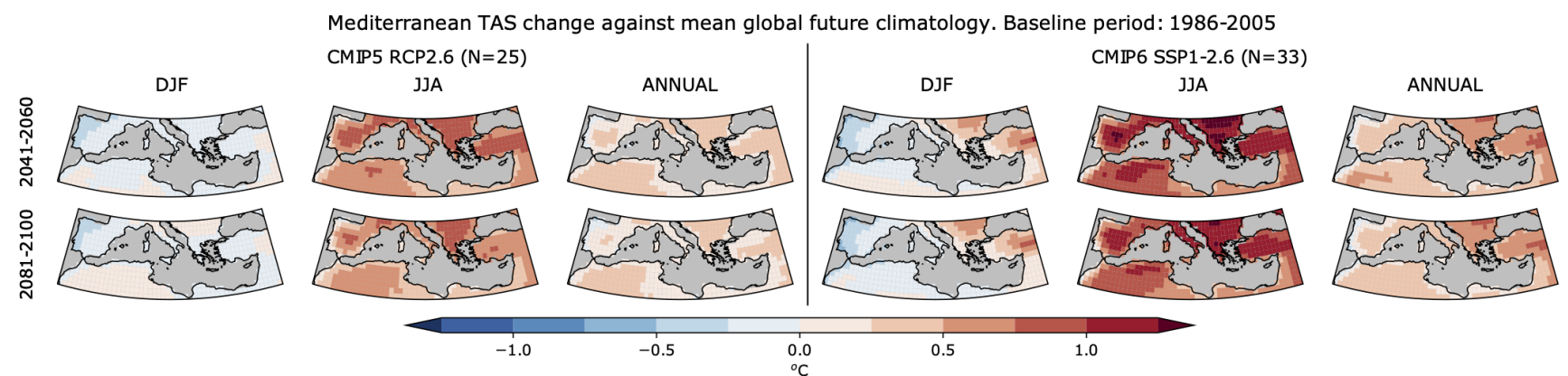

Mediterranean PR change against mean $30^{\circ} \mathrm{N}-45^{\circ} \mathrm{N}$ latitudinal belt future climatology. Baseline period: $1986-2005$

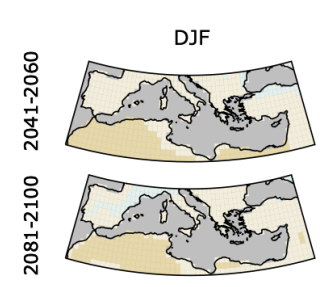
CMIP5 RCP2.6 $(\mathrm{N}=25)$
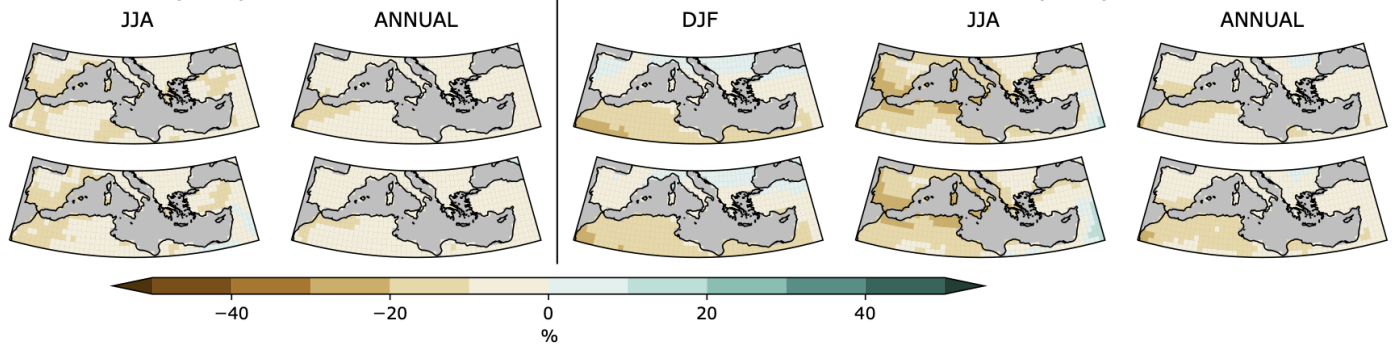

Figure S1. same as Fig. 1 but for scenario 2.6 
Mediterranean vs $30^{\circ} \mathrm{N}-45^{\circ} \mathrm{N}$ latitudinal belt DJF precipitation. $10 y r$ rolling means 1960-2100, Baseline: 1986-2005
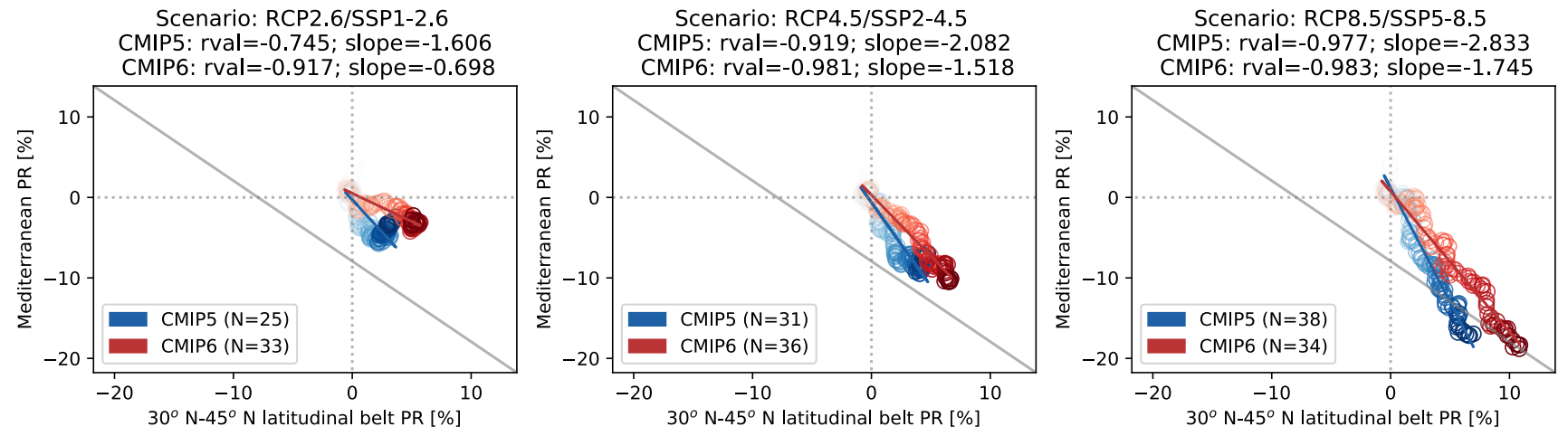

Mediterranean vs $30^{\circ} \mathrm{N}-45^{\circ} \mathrm{N}$ latitudinal belt JJA precipitation.

10yr rolling means 1960-2100, Baseline: 1986-2005
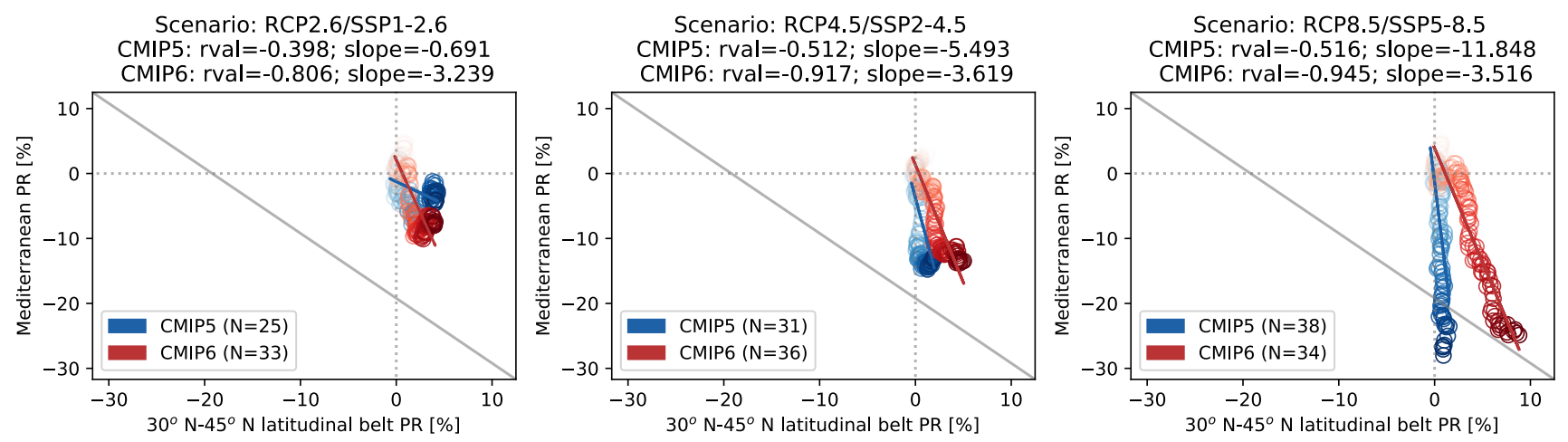

Figure S2. Same as Fig. 2 but plotting the Mediterranean precipitation change against the $30^{\circ} \mathrm{N}-45^{\circ} \mathrm{N}$ latitudinal belt precipitation change. 
RCP8.5/SSP5-8.5 JJA precipitation change. Baseline: 1986-2005

CMIP5 2021-2040 ( $\mathrm{N}=38)$

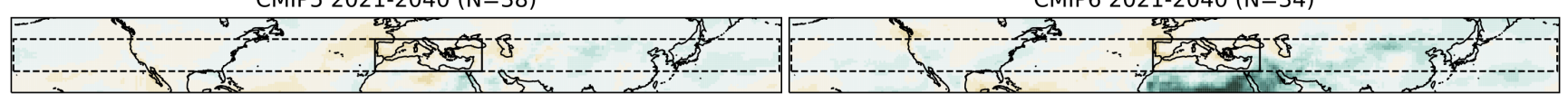

CMIP5 2061-2080 ( $\mathrm{N}=38)$

CMIP6 2061-2080 ( $\mathrm{N}=34)$

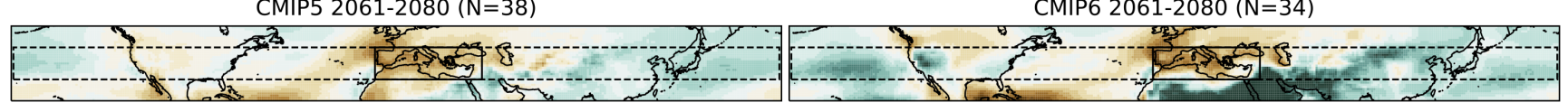

CMIP5 2081-2100 ( $\mathrm{N}=38)$

CMIP6 2081-2100 ( $\mathrm{N}=34)$
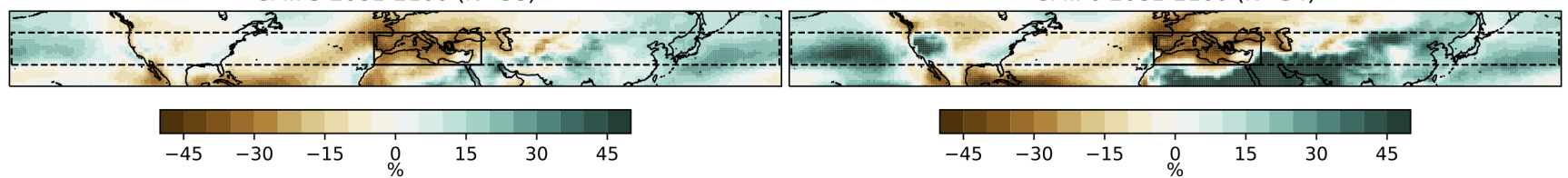

Figure S3. Summer precipitation differences for the future periods 2021-2040, 2061-2080 and 2081-2100 with respect to the baseline period 1986-2005, as projected by CMIP5 (left) and CMIP6 (right) multi-model means. The dashed line encloses the $30^{\circ} \mathrm{N}-45^{\circ} \mathrm{N}$ latitudinal belt and the solid line the Mediterranean region. 
Mediterranean DJF precipitation vs global DJF temperature.

$10 y r$ rolling means 1960-2100, Baseline: 1986-2005

Scenario: RCP2.6/SSP1-2.6

CMIP5: $r$ val $=-0.943$; slope $=-4.305$

CMIP6: rval $=-0.951$; slope $=-2.233$

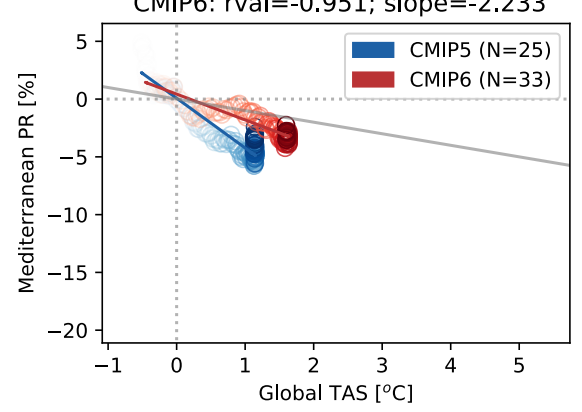

Scenario: RCP4.5/SSP2-4.5

CMIP5: rval=-0.976; slope $=-4.449$

CMIP6: $r$ al $=-0.988$; slope $=-3.690$

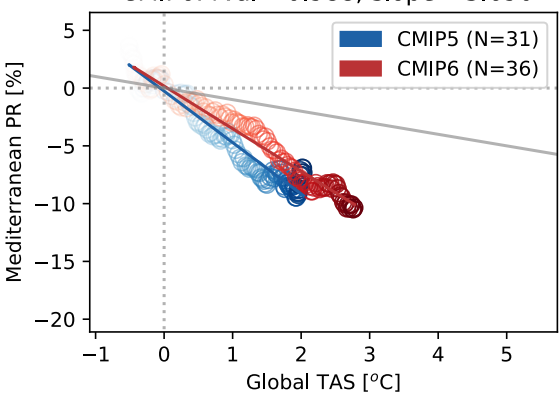

Scenario: RCP8.5/SSP5-8.5

CMIP5: $r$ val $=-0.992$; slope $=-4.343$ CMIP6: $r$ al $=-0.992 ;$ slope $=-3.746$

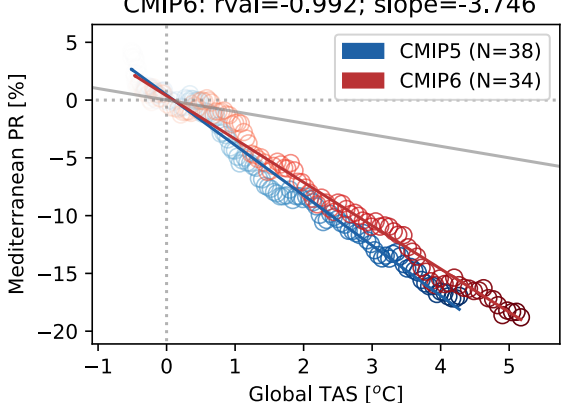

Mediterranean JJA precipitation vs global JJA temperature.

10yr rolling means 1960-2100, Baseline: 1986-2005
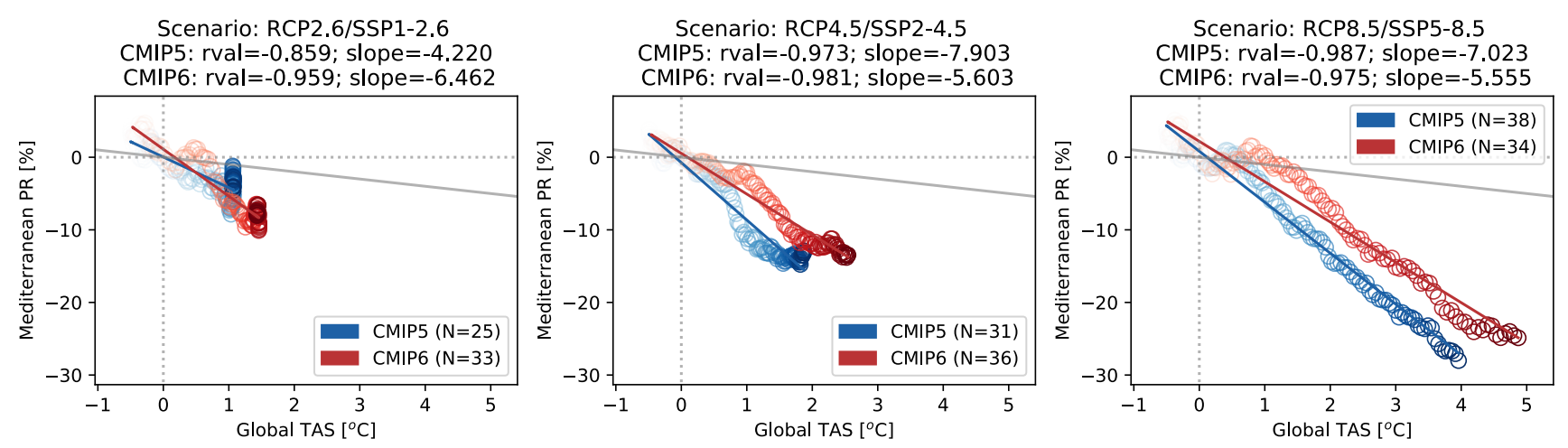

Figure S4. Same as Fig. 2 but plotting the Mediterranean precipitation change against the global temperature change. 
(a) DJF Mediterranean region historic trends and changes for a future scenario
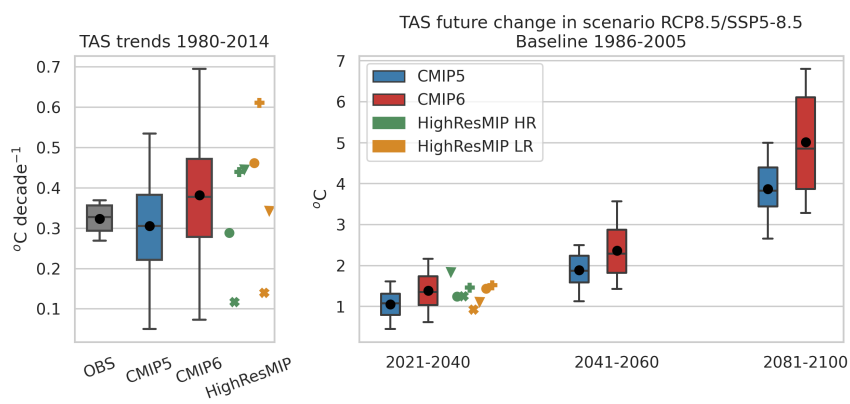

(c) JJA Mediterranean region historic trends and changes for a future scenario
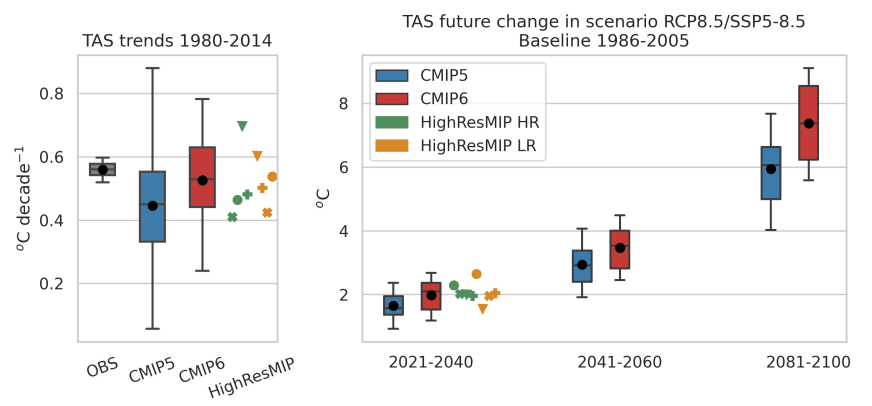

(b) DJF Mediterranean region historic trends and changes for a future scenario
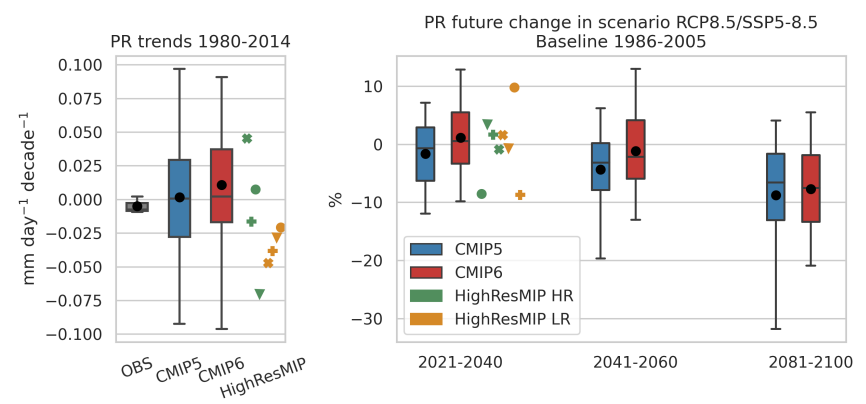

(d) JJA Mediterranean region historic trends and changes for a future scenario
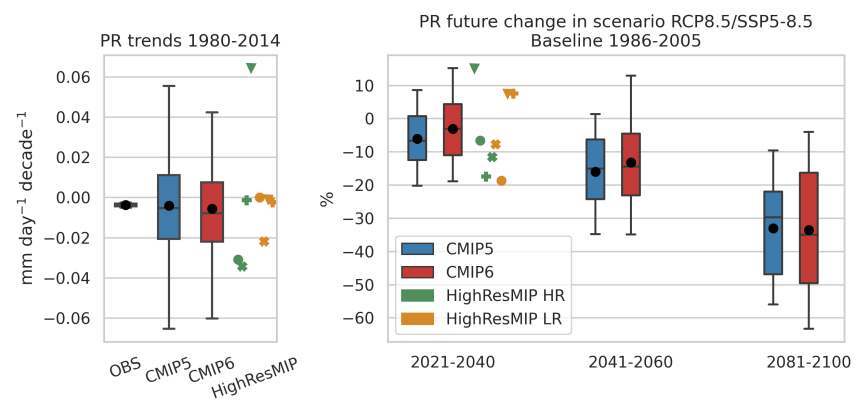

Figure S5. Winter (a, b) and summer (c, d) temperature (a, c) and precipitation (b, d) historical trends of the observational ensemble, CMIP5, CMIP6 and HighResMIP (left panels of the sub-figures), and projected changes for the near, mid and long-term periods with respect to the baseline period (right panels of the sub-figures). In the box plots, the black horizontal line represents the median and the black dot is the mean. The interquartilic range (IQR) and whiskers are defined by the 25th-75th and 5th-95th percentiles, respectively. HighResMIP models are displayed as markers, enabling for a comparison of the HR (red) and LR (blue) models within the experiment. The same markers are used for two different resolution runs of the same model (see Table S1)

Table S1. HighResMIP markers used in Fig. S5 to highlight differences in resolution from the same model.

\begin{tabular}{ll}
\hline Marker & LR/HR Models \\
\hline $\boldsymbol{\nabla}$ & CMCC-CM2-SR5 / CMCC-CM2-VHR4 \\
$\boldsymbol{*}$ & CNRM-CM6-1 / CNRM-CM6-1-HR \\
$\boldsymbol{+}$ & EC-Earth3P / EC-Earth3P-HR \\
$\boldsymbol{\bullet}$ & HadGEM3-GC31-LL / HadGEM3-GC31-HM \\
\hline
\end{tabular}


CMIP5 \& CMIP6 TAS changes over JJA \& DJF Baseline period: 1986-2005

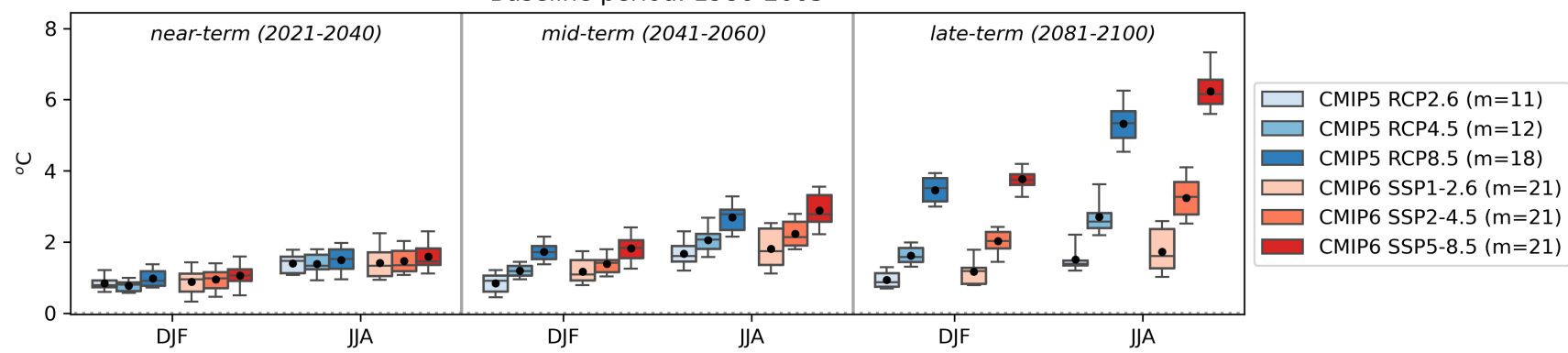

Figure S6. Same as Fig. 3a but computed with a subset distribution of CMIP5 and CMIP6 members with ECS between 2.6 and 3.3

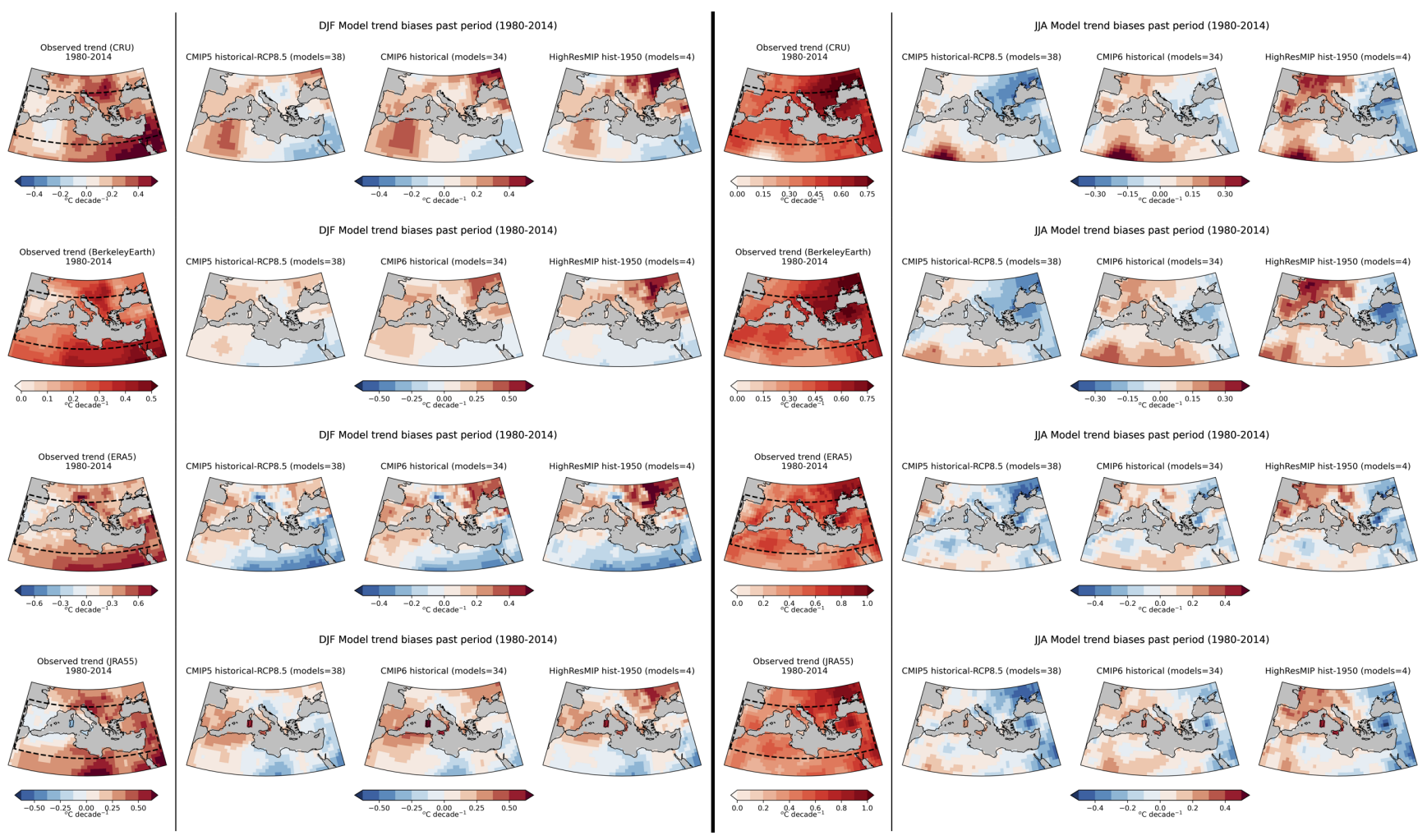

Figure S7. Historical period TAS trend biases against observational references (rows) for CMIP5, CMIP6 and HighResMIP multi-model means during winter (left) and summer (right). 

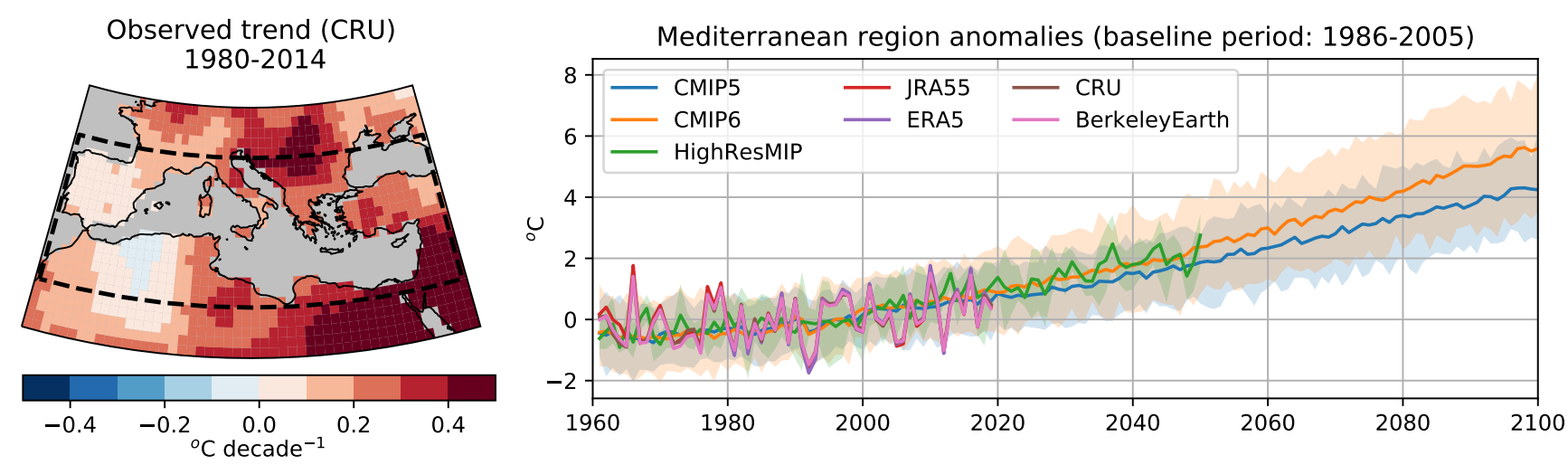

DJF Model temperature change (2021-2040)

CMIP5 RCP8.5 (models $=38$ )

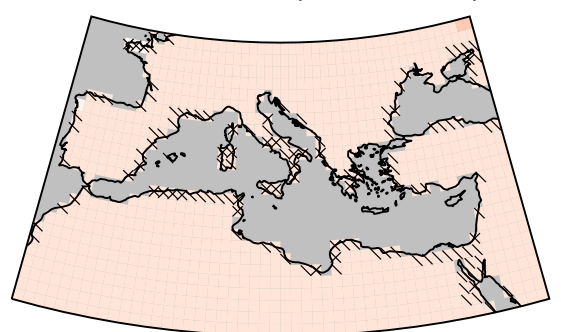

CMIP6 SSP5-8.5 (models $=34$ )

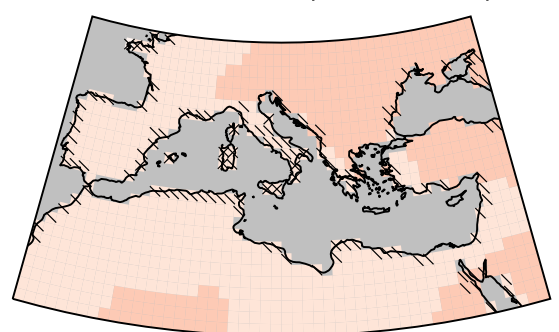

HighResMIP highres-future (models $=4$ )

DJF Model temperature change (2041-2060)

CMIP5 RCP8.5 (models $=38$ )

CMIP6 SSP5-8.5 (models $=34$ )
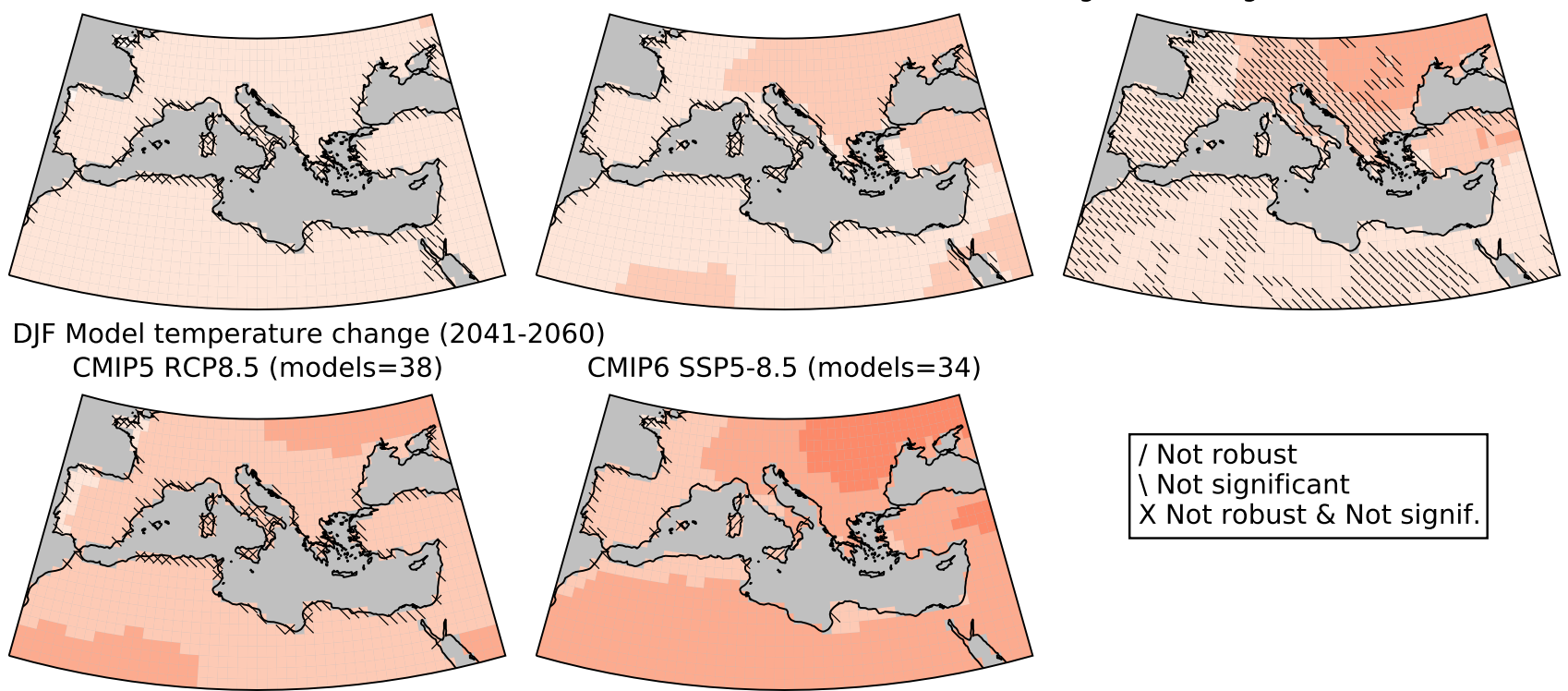

DJF Model temperature change (2081-2100)

CMIP5 RCP8.5 (models $=38$ )

CMIP6 SSP5-8.5 (models $=34$ )
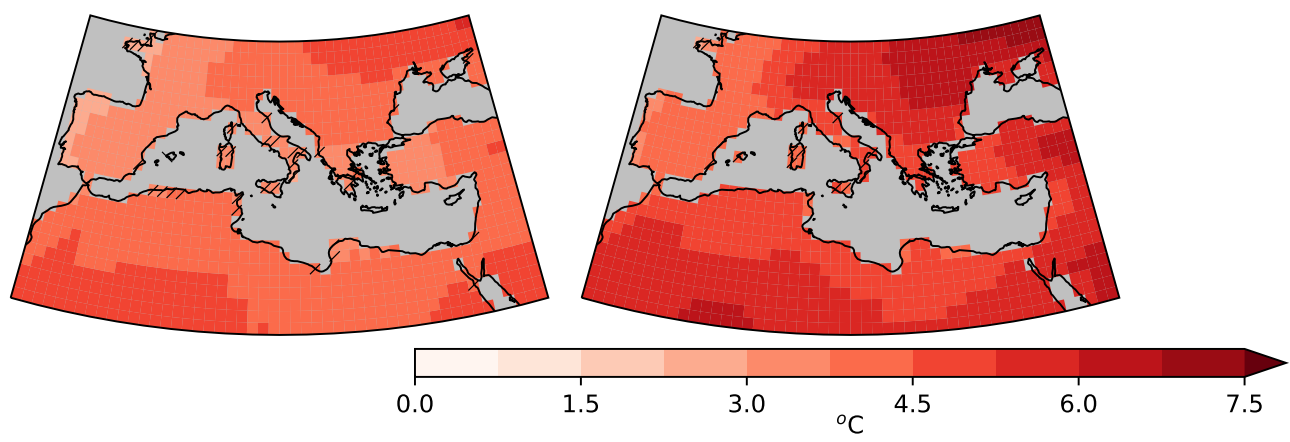

Figure S8. Same as Fig. 4 but for winter 
Observed trend (CRU) 1980-2014

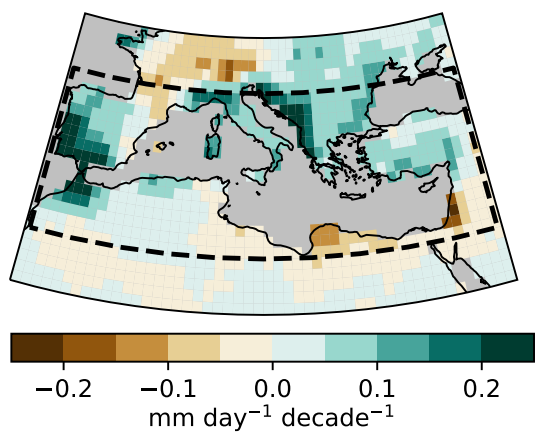

DJF Model precipitation change (2021-2040) CMIP5 RCP8.5 (models $=38$ )

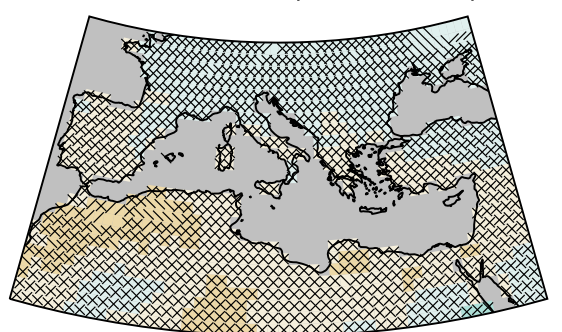

CMIP6 SSP5-8.5 (models $=34$ )

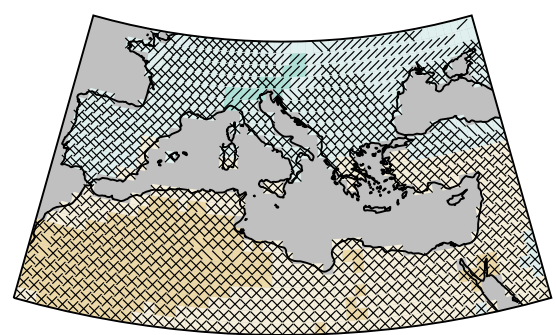

DJF Model precipitation change (2041-2060)

CMIP5 RCP8.5 (models=38)
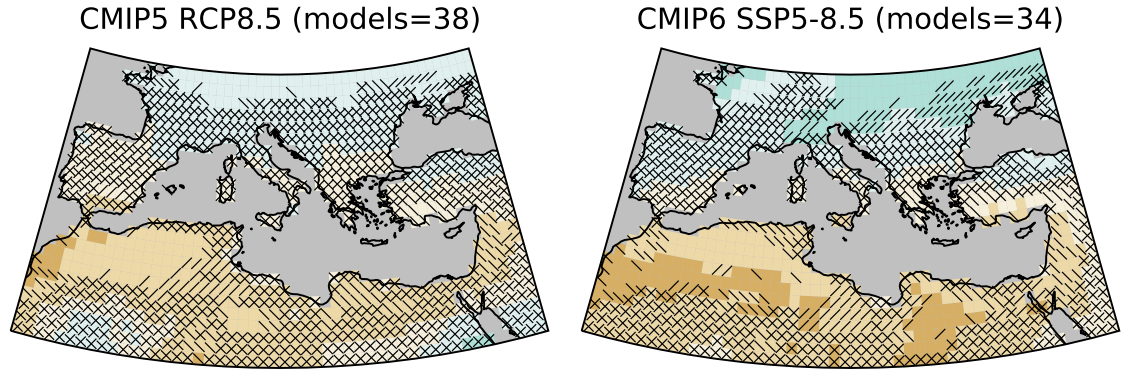

DJF Model precipitation change (2081-2100) CMIP5 RCP8.5 (models $=38$ )
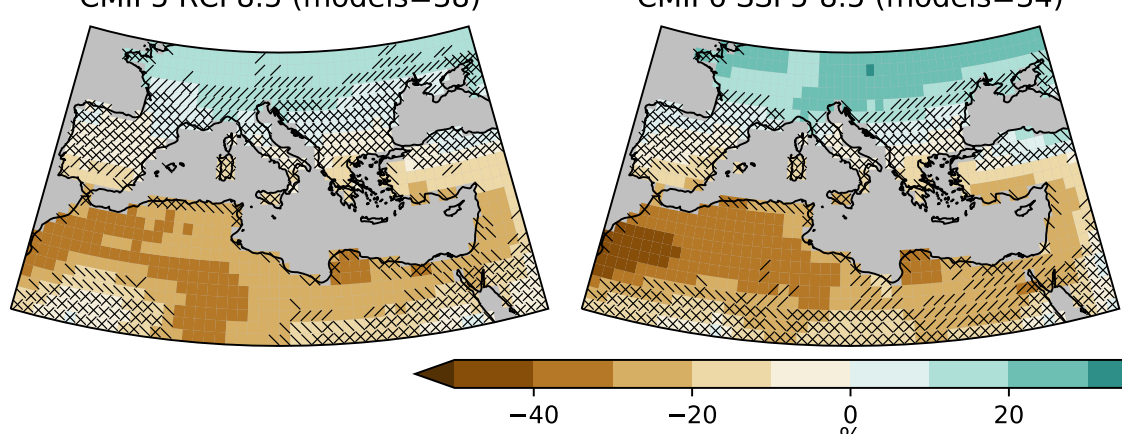

HighResMIP highres-future (models $=4$ )

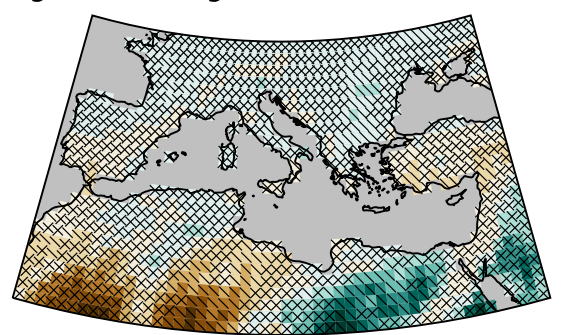

/ Not robust

$\backslash$ Not significant

$X$ Not robust $\&$ Not signif.

Figure S9. Same as Fig. 5 but for winter 


\section{Model performance over the historical period}

The ensembles used in the present study contain many models and they all perform differently for different diagnostics. Fig. S10 shows the normalised RMSE from diverse diagnostics (rows) of all CMIP6 models (columns) for an historical period (1980-2014). The values are normalised by first subtracting the median RMSE of the single models to each model's RMSE,

5 and secondly, dividing by the median RMSE (Gleckler et al., 2008) The boxes are diagonally split to show RMSE against JRA55 and CRU observational datasets. Negative values indicate a better than median performance, positive values worse than median performance.

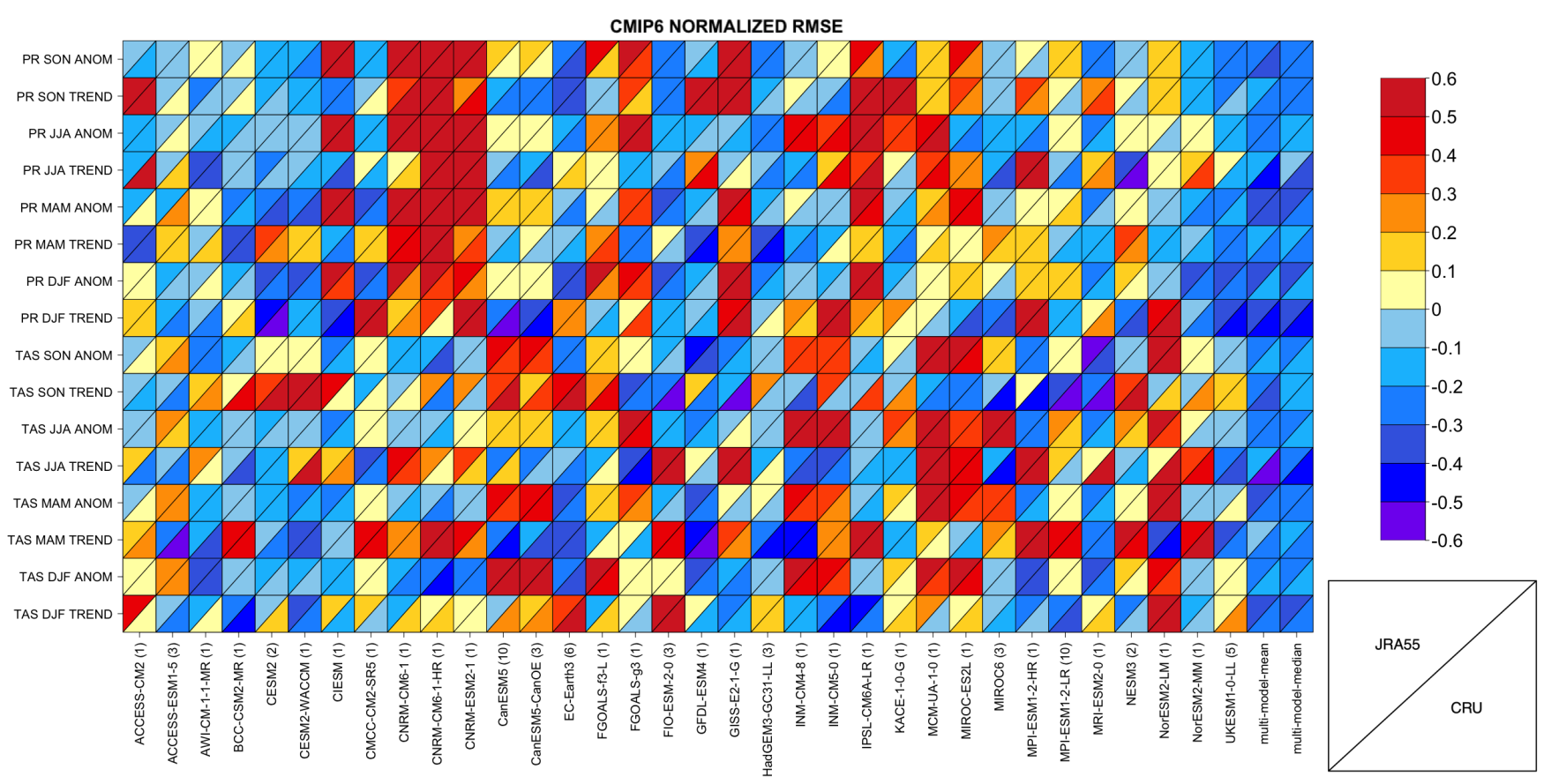

Figure S10. Seasonal normalized root mean squared error of TAS and PR anomalies and trends over the Mediterranean (rows) between each CMIP6 model (columns) and observational references for 1980-2014. Each cell is divided in two triangles to display the RMSE against JRA55 and CRU. The last two columns show the RMSE of multi-model ensemble mean and median.

Each models' performance is very different along the diagnostics. Some models perform better over most of the diagnostics, some seem to perform well for some diagnostics or variables and bad for others, and some perform worse than the RMSE median for most diagnostics. There are models that apparently share characteristics as some diagnostics yield similar results for models from CCCma and CNRM-CERFACS. Differences between models from the same institutions usually are the grid resolution, the ocean model or the chemistry of both the atmosphere and ocean, to name a few (ref*). The potential dependency between models raises another concern when dealing with multi-model ensemble projections. Dependent models bear the risk of producing unbalanced mean projections as more weight could be given to a certain way of modelling the climate. 

median. Showing that while there are models that perform better than the ensemble mean, it is still better to use the mean than single models when considering multiple diagnostics. The fact that the multi-model ensemble performance is consistently better for all diagnostics shows how single model errors partly cancel off (Tebaldi and Knutti, 2007). Arriving at a better representation of the climate by combining different models is one of the pillars behind the Coupled Model Intercomparison Project.

The comparison between the CMIP5, CMIP6 and HighResMIP models is shown in Fig. S2.10S11. The multi-model means from CMIP5 and CMIP6 show very similar RMSE values, on average, across diagnostics. The HighResMIP ensemble is too small to compare it's multi-model mean to both CMIPs.
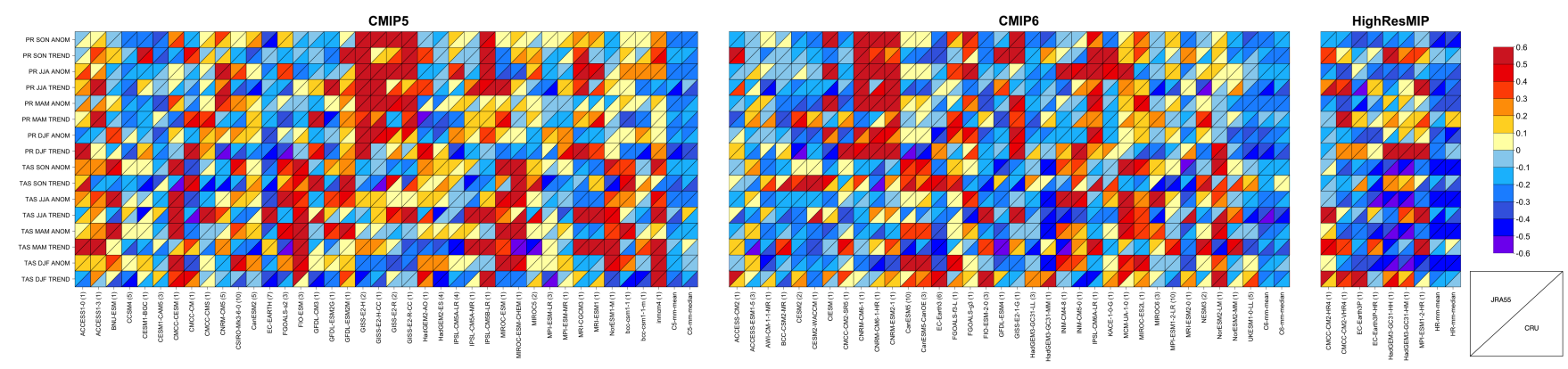

Figure S11. Same as Fig. S10 but normalising RMSE using the complete ensemble of models from CMIP5, CMIP6 and HighResMIP. 


\section{References}

25 Gleckler, P. J., Taylor, K. E., and Doutriaux, C.: Performance metrics for climate models, Journal of Geophysical Research Atmospheres, 113, https://doi.org/10.1029/2007JD008972, 2008.

Tebaldi, C. and Knutti, R.: The use of the multi-model ensemble in probabilistic climate projections, Ocean Model., 365, 2053-2075, https://doi.org/10.1098/rsta.2007.2076, 2007. 